\begin{tabular}{|c|c|}
\hline \multicolumn{2}{|c|}{ Statistica Sinica Preprint No: SS-2021-0060 } \\
\hline Title & $\begin{array}{l}\text { High-Dimensional Asymptotic Behavior of Inference } \\
\text { Based on Gwas Summary Statistics }\end{array}$ \\
\hline Manuscript ID & SS-2021-0060 \\
\hline URL & http://www.stat.sinica.edu.tw/statistica/ \\
\hline DOI & $10.5705 /$ ss.202021.0060 \\
\hline Complete List of Authors & $\begin{array}{l}\text { Jiming Jiang, } \\
\text { Wei Jiang, } \\
\text { Debashis Paul, } \\
\text { Yiliang Zhang and } \\
\text { Hongyu Zhao }\end{array}$ \\
\hline Corresponding Author & Jiming Jiang \\
\hline E-mail & jimjiang@ucdavis.edu \\
\hline
\end{tabular}


Statistica Sinica

\title{
High-dimensional Asymptotic Behavior of Inference Based on GWAS Summary Statistics
}

Jiming Jiang, Wei Jiang, Debashis Paul, Yiliang Zhang and Hongyu Zhao

\author{
University of California, Davis and Yale University
}

Abstract: We study high-dimensional asymptotic behavior of inference based on summary statistics that are widely used in genome-wide association studies (GWAS) under model misspecification. The high dimensionality is in the sense that the number of single-nucleotide polymorphisms (SNPs) under consideration may be much larger than the sample size. The model misspecification is in the sense that the number of causal SNPs may be much smaller than the total number of SNPs under consideration. Specifically, consistency and asymptotic normality of estimators of the heritability and genetic covariance, two parameters of genetic interest, are established. The theoretical results are supported by empirical studies involving simulated and real data.

Key words and phrases: asymptotic normality, Bernoulli, consistency, genetic covariance, heritability, martingale, model misspecification, random matrix theory 


\section{Introduction}

Over the past fifteen years, genome-wide association studies (GWAS) have identified tens of thousands of single-nucleotide polymorphisms (SNPs) associated with complex human traits and diseases (Buniello et al. (2019) $)$. Besides the success in finding risk loci, the estimation of heritability and genetic covariance based on collected GWAS data also provides insights into the genetic basis of complex traits/diseases (Tenesa and Haley (2013); van Rheenen et al. (2019)). Heritability is the proportion of phenotypic variance due to genetic effects while genetic covariance is the covariance of genetic effects contributing to two phenotypes. Methods based on linear mixed model (LMM) in conjunction with the restricted maximum likelihood (REML) algorithm have been developed to estimate these two quantities of significant genetic interests (Yang et al. (2010); Lee et al. (2012)). Compared with traditional family-based approaches for estimating these two quantities, these methods do not need to collect related samples and can use large GWAS samples for estimation. Moreover, they do not require the studied phenotypes measured on the same individuals when estimating genetic covariance, which makes it possible to study a spectrum of human complex traits/diseases simultaneously by using different cohorts. As for the statistical properties of these estimates, high-dimensional asymptotic 
theory of REML for heritability estimation has been recently established even under a misspecified LMM, which provides theoretical support for the robustness of the REML estimator (Jiang et al. (2016)).

However, methods based on LMM have only gained middling popularity because they require individual-level genotype and phenotype data, which are usually difficult to obtain owing to policy and privacy concerns. Increasingly accessible marginal association statistics from GWAS and advances in analytical methods relying only on these summary statistics, have circumvented challenges in data sharing and greatly accelerated research in complex trait/disease genetics. Due to its computational efficiency, linkage disequilibrium (LD) score regression (LDSC; Bulik-Sullivan et al. $(2015 \mathrm{a}$ b) $)$ is currently the most popular method for estimating heritability and genetic covariance using GWAS summary statistics. Based on this method, bioinformatics servers have been built to improve the computation as well as visualization of heritability and genetic covariance for a wide range of phenotypes (Zheng et al. (2017)).

In a typical GWAS data set, the total number of SNPs, $p$ (e.g., $10^{6} \sim$ $10^{7}$ ), is often much larger than the sample size, $n$ (e.g., $10^{3} \sim 10^{6}$ ), that is, $p \gg n$. In addition, more SNPs can be observed when more subjects are recruited in GWAS, i.e. $p$ increases with $n$. In other words, GWAS data 
analysis has a nature of high-dimensionality. Despite the polygenicity of many phenotypes, such as anthropometric characteristics (Berndt et al. (2013)) and psychiatric disorders (Sullivan, Daly and O'donovan (2012)), the SNPs having biological effects on the phenotypes (causal SNPs) are still only a small portion of the total SNPs. However, summary-statistics-based heritability or genetic covariance estimation methods, such as LDSC, often assume that the effects of all SNPs are nonzero while the true underlying model might be sparse. Namely, the assumed model is misspecified in LDSC. Although LDSC has become a routine for post-GWAS analysis to estimate heritability and genetic covariance, the high-dimensional asymptotic behavior of LDSC under model misspecification has not yet been rigorously justified. Therefore, there is a pressing need for a theoretical justification for LDSC. In this paper, we establish consistency and asymptotic normality of the heritability and genetic covariance eatimators from LDSC, in the regime of high-dimensional statistics as both the sample size $n$ and the dimension of the random effects $p$ tend to infinity. Our results indicate that the misspecified LDSC estimators converge to the desired true values of the genetic quantities and we also provide their convergence rate (in probability) as well as asymptotic variances. Our theoretical results are fully supported by our empirical studies. 
1.1 LDSC estimation under model misspecification

\subsection{LDSC estimation under model misspecification}

We first introduce how to estimate heritability using LDSC and GWAS summary statistics (Bulik-Sullivan et al. (2015a)). Built upon LMM, phenotypes are modeled as

$$
\phi=X \beta+\epsilon,
$$

where $\phi$ is an $n \times 1$ vector of (quantitative) phenotypes, $X$ is an $n \times p$ random design matrix of genotypes normalized to mean zero and variance one, $\beta$ is a $p \times 1$ vector of random effects following the $N\left[0,\left(h^{2} / p\right) I_{p}\right]$ distribution, in which $I_{p}$ denotes the $p$-dimensional identity matrix, and $\epsilon$ is an $n \times 1$ vector of errors that is distributed as $N\left[0,\left(1-h^{2}\right) I_{n}\right]$. Here $X, \beta$, and $\epsilon$ are mutually independent. We further assume the genotypes of different subjects are independent from each other. Before the normalization, the genotypes are coded as 0,1 , and 2, which are the allelic dosages (number of minor alleles) of the variants. Denote $f_{j}$ as the known minor allele frequency (MAF) of SNP $j$. According to the Hardy-Weinberg equilibrium (HWE), the probabilities of the genotype being 0,1 , and 2 for SNP $j$ are $(1-$ $\left.f_{j}\right)^{2}, 2 f_{j}\left(1-f_{j}\right)$, and $f_{j}^{2}$, respectively. Thus, after normalization, we have $-2 f_{j} / \sqrt{2 f_{j}\left(1-f_{j}\right)},\left(1-2 f_{j}\right) / \sqrt{2 f_{j}\left(1-f_{j}\right)}$, and $\left(2-2 f_{j}\right) / \sqrt{2 f_{j}\left(1-f_{j}\right)}$ in 
1.1 LDSC estimation under model misspecification

$X$ accordingly. It follows that $\mathrm{E}\left(X X^{\prime}\right)=p I_{n}$, hence

$$
\operatorname{Var}(\phi)=\operatorname{Var}(X \beta)+\operatorname{Var}(\epsilon)=\frac{h^{2}}{p} \mathrm{E}\left(X X^{\prime}\right)+\left(1-h^{2}\right) I_{n}=I_{n} .
$$

The heritability is defined as the proportion of phenotypic variance attributed to genetic factors. Based on this definition, the heritability of the phenotype is the sum of the random effect variances, which is $h^{2}$.

Due to the existence of LD, genotypes of different SNPs are correlated especially for SNPs located nearby (Stephens et al. (2001)). We denote $r_{j k}$ as the genotypic correlation between $\operatorname{SNP} j$ and SNP $k$, that is, $r_{j k}=$ $\mathrm{E}\left(X_{i j} X_{i k}\right)$, which does not depend on $i$. The pairwise correlations among SNPs are stored in an LD matrix $R$, i.e. for any subject $i, 1 \leq i \leq n$, $\operatorname{cov}\left(X_{[i]}\right)=R$, where $X_{[i]}$ is the $i$ th row of $X$. The correlations usually decay with the increase of pairwise distances and hence the $\operatorname{LD}$ matrix is $C$ dependent overall (to be detailed below). The LD score of a SNP is defined by $l_{j}=\sum_{k=1}^{p} r_{j k}^{2}$, where the sum is taken over all the variants including SNP $j$ itself $\left(r_{j j}=1\right)$. As a special case, when SNP $j$ is independent with other SNPs, we have $l_{j}=1$. In practice, the LD matrix and LD scores can be obtained from a public external reference panel constituting individual-level genotype data (e.g. 1000 Genomes Project Clarke et al. |(2017)). Following the arguments in Bulik-Sullivan et al. (2015a), $r_{j k}^{2}$ in the definition of $l_{j}$ is replaced by the approximately unbiased estimator 
1.1 LDSC estimation under model misspecification

given by $r_{j k, a d j}^{2}=\hat{r}_{j k}^{2}-\left(1-\hat{r}_{j k}^{2}\right) /(N-2)$, where $N$ is the sample size of the reference panel and $\hat{r}_{j k}^{2}$ denotes the square of the sample Pearson correlation coefficient.

The design matrix $X$ may be difficult to access due to privacy and security issues. The merit of LDSC is that it only needs more accessible GWAS summary statistics as the input. In GWAS summary statistics, we have $z$-score of each SNP which reflects the marginal association between the phenotype and the SNP. Since the marginal heritability explained by one SNP is usually small, the z-score of SNP $j z_{j}$ can be approximated by $z_{j}=X_{j}^{\prime} \phi / \sqrt{n}$, where $X_{j}$ denotes the $j$ th column of $X$. According to LDSC (Bulik-Sullivan et al. (2015b)), the heritability can be estimated by solving the following linear regression:

$$
\mathrm{E}\left(z_{j}^{2}\right)=1+h^{2}(n / p) l_{j}, \quad j=1, \ldots, p
$$

where the intercept is fixed as 1 .

The model has been generalized to estimate genetic covariance between phenotypes (Bulik-Sullivan et al. (2015a)). Genetic covariance analysis can provide new insights into the shared genetics of many phenotypes, and has a variety of downstream applications van Rheenen et al. (2019), Zhang et al. (2021)), which has become a popular post-GWAS analysis tool. Let us assume that there are two GWAS for two different phenotypes 
1.1 LDSC estimation under model misspecification

with sample sizes $n_{1}$ and $n_{2}$, respectively. The two GWAS share the same set of $p$ SNPs but are not necessarily performed within the same cohort. In practice, two different GWAS may share a subset of subjects. Denote the number of shared subjects as $n_{\mathrm{o}}$ (the subscript o refers to "overlap"), $0 \leq n_{\mathrm{o}} \leq n_{1} \wedge n_{2} \equiv \min \left(n_{1}, n_{2}\right)$. The phenotypes are modeled as

$$
\begin{aligned}
& \phi_{1}=X \beta+\epsilon \\
& \phi_{2}=Y \gamma+\delta,
\end{aligned}
$$

where $\phi_{1}$ and $\phi_{2}$ are $n_{1} \times 1$ and $n_{2} \times 1$ vectors of phenotypes, $\mathrm{X}$ and $\mathrm{Y}$ are $n_{1} \times p$ and $n_{2} \times p$ random design matrices of genotypes normalized to mean zero and variance one with the same $\mathrm{LD}$ matrix, $\beta$ and $\gamma$ are two $p \times 1$ vectors of random effects jointly normally distributed so that $\mathrm{E}(\beta)=\mathrm{E}(\gamma)=0$ and

$$
\operatorname{Var}\left(\begin{array}{l}
\beta \\
\gamma
\end{array}\right)=\frac{1}{p}\left(\begin{array}{ll}
h_{1}^{2} I_{p} & \rho_{g} I_{p} \\
\rho_{g} I_{p} & h_{2}^{2} I_{p}
\end{array}\right),
$$

and $\epsilon$ and $\delta$ are $n_{1} \times 1$ and $n_{2} \times 1$ vectors of random errors. The marginal distributions of $\epsilon$ and $\delta$ are $N\left[0,\left(1-h_{1}^{2}\right) I_{n_{1}}\right]$ and $N\left[0,\left(1-h_{2}^{2}\right) I_{n_{2}}\right]$, respectively. Here, $(X, Y),(\beta, \gamma)$, and $(\epsilon, \delta)$ are independent. Without loss of generality, we assume the first $n_{\mathrm{o}}$ samples in each study are shared. $\epsilon$ and $\delta$ are correlated due to the non-genetic correlation introduced through the 
1.1 LDSC estimation under model misspecification

overlapping samples:

$$
\operatorname{cov}\left(\epsilon_{i}, \delta_{j}\right)=\left\{\begin{array}{ll}
\rho_{\mathrm{e}}, & 1 \leq i=j \leq n_{\mathrm{o}} \\
0, & \text { otherwise }
\end{array} .\right.
$$

Similar to 1.3$)$, to estimate the genetic covariance, $\rho_{\mathrm{g}}$, one can fit the following linear regression model:

$$
\mathrm{E}\left(z_{1 j} z_{2 j}\right)=\rho n_{\mathrm{o}} / \sqrt{n_{1} n_{2}}+\rho_{\mathrm{g}}\left(\sqrt{n_{1} n_{2}} / p\right) l_{j}, \quad j=1, \ldots, p
$$

where $\rho=\rho_{\mathrm{g}}+\rho_{\mathrm{e}}$. As a special case, if study 1 and study 2 are the same study, which means that we have $n_{1}=n_{2}=n_{\mathrm{o}}, \rho_{\mathrm{g}}=h_{1}^{2}=h_{2}^{2}=h^{2}$, and $\rho_{\mathrm{e}}=1-h^{2}$, then model 1.5 reduces to model 1.3$)$.

A basic assumption in the above introduced LMMs is that all SNPs contribute to the phenotypic variance. In reality, however, only a subset of the SNPs are causal SNPs. Let $S, T_{1}, T_{2} \subset\{1,2, \ldots, p\}$ represent the indices of causal SNPs shared in both traits, those only presented in trait 1, and those only presented in trait 2 , respectively. In other words, $S \cup T_{k}$ are the indices of causal SNPs for trait $k$ (where $k=1,2$ ). Note that $S, T_{1}$ and $T_{2}$ are mutually exclusive subsets. Let $\beta_{S}$ and $\gamma_{S}$ be the vectors of random effects corresponding to the SNPs in $S$ for both phenotypes. Similarly, $\beta_{T_{k}}$ and $\gamma_{T_{k}}$ are defined as the random effect vectors corresponding to the SNPs in $T_{k}, k=1,2$. Let $m=|S|$ (cardinality), $m_{1}=\left|T_{1}\right|$, and $m_{2}=\left|T_{2}\right|$. Under 
1.1 LDSC estimation under model misspecification

the true model, the distribution of $\beta_{j}$ is $N\left[0, h_{1}^{2} /\left(m+m_{1}\right)\right]$ for $j \in S \cup T_{1}$ and $\beta_{j}=0$ for $j \notin S \cup T_{1}$. Similarly, we have $\gamma_{j} \sim N\left[0, h_{2}^{2} /\left(m+m_{2}\right)\right]$ when $j \in S \cup T_{2}$ and $\gamma_{j}=0$ when $j \notin S \cup T_{2}$. The true LMMs can then be expressed as

$$
\begin{aligned}
\phi_{1} & =X_{S} \beta_{S}+X_{T_{1}} \beta_{T_{1}}+\epsilon \\
\phi_{2} & =Y_{S} \gamma_{S}+Y_{T_{2}} \gamma_{T_{2}}+\delta
\end{aligned}
$$

[compare to $(1.4)]$, where $X_{A}$ is the normalized genotype matrix for the SNPs in set $A$ (where $A=S$ or $T_{1}$ ) and $Y_{A}$ is defined similarly ( $A=S$ or $T_{2}$ ). The joint distribution for the effects of the SNPs in $S$ is given by

$$
\left(\begin{array}{c}
\beta_{S} \\
\gamma_{S}
\end{array}\right) \sim N\left[\left(\begin{array}{c}
0 \\
0
\end{array}\right),\left(\begin{array}{cc}
\frac{h_{1}^{2}}{m+m_{1}} I_{m} & \frac{\rho_{g}}{m} I_{m} \\
\frac{\rho_{g}}{m} I_{m} & \frac{h_{2}^{2}}{m+m_{2}} I_{m}
\end{array}\right)\right] .
$$

Here, $h_{1}^{2}, h_{2}^{2}$, and $\rho_{\mathrm{g}}$ are the heritability of phenotype 1 , heritability of phenotype 2, and genetic covariance between phenotype 1 and 2 under the true model, respectively. Detailed assumptions concerning the distributions of genotype matrices and random effects under the true model are given in the sequel.

In practice, it is impossible to determine whether a SNP is causal for the phenotype. Therefore, we have to follow the assumption in LDSC that all of the SNPs are causal in order to estimate heritability and genetic covariance, 
1.2 Numerical illustrations

which actually leads to the misspecified model. The main goal of this paper is to show that the consistency and asymptotic normality properties of the misspecified LDSC estimators are valid when $n, p$ and $m$ tend to infinity.

We conclude this section with a couple of numerical illustrations.

\subsection{Numerical illustrations}

In GWAS, SNPs are high-density bi-allelic genetic markers. Each SNP can be considered as a binomial random variable with two trials and the probability of "success" is the minor allele frequency $f_{j}$. In each sample, the SNP genotypes are correlated, which is known as LD. To mimic the LD matrix of human genome, the LD matrix we used to simulate genotype data has a block structure, which is a special case of the $C$-dependent relationship to be formally introduced later. In this simulation, since the LD matrix is known to us, we directly calculate LD scores based on the true LD matrix. Please note that we usually rely on external reference panel to estimate LD scores in practice due to unavailability of true LD matrix. Later we will discuss the effect of the randomness in the estimation of LD scores. According to the genome partition software LDetect Berisa and Pickrell (2016)), there are $\sim 2,000$ independent blocks for $\sim 5,000,000$ SNPs in the human genome of European ancestry. We randomly selected 200 blocks 
1.2 Numerical illustrations

(3 blocks were further removed due to small size), and scaled down the number of SNPs in each block to make the total number of SNPs 20,000. In the following illustrative examples, the number of SNPs in each block ranges from 1 to 502 with mean 102. The SNP genotypes between different blocks are independent. We further assume that the local LD matrix for each block follows $\mathrm{AR}(1)$ structure, that is, if $\mathrm{SNP} j$ and $\mathrm{SNP}(j+d)$ are in the same block, the genotypic correlation between these two SNPs is $\alpha^{d}$. We use the $\mathrm{AR}(1)$ correlation structure here to mimic the observation that LD decays with the distance in a real genome. The $\mathrm{AR}(1)$ model coefficient $\alpha$ for each block is independently sampled from $\{0.1, \ldots, 0.9\}$ with equal probability. After an LD matrix was generated, we fixed it throughout the entire illustrative experiment. In our simulations, we set the SNPs in the same block sharing the same MAF, which is sampled from the Uniform $(0.05,0.5)$ distribution. CorBin is a highly efficient R-package to generate high-dimensional binary/binomial data with specified correlation structures, including exchangeable, $\mathrm{AR}(1)$ and K-dependent (Jiang et al. ॥(2020)). We used CorBin to generate correlated genotype data for each individual.

Heritability. In this illustrative simulation for heritability estimation, we fixed $p=20,000$ and true value of heritability $h^{2}=0.6$. Let $b_{j}=$ 
1.2 Numerical illustrations

1 if SNP $j$ is causal, whose corresponding effect size follows $N\left(0, h^{2} / m\right)$; otherwise, $b_{j}=0$ and the effect of SNP $j$ is zero. The indicators $b_{1}, \ldots, b_{p}$ are independent Bernoulli random variables such that $\mathrm{P}\left(b_{j}=1\right)=\omega \in$ $(0,1)$. Note that $m=\sum_{j=1}^{p} b_{j}$. We use $\tau$ to represent the ratio of sample size to SNP number (i.e., $\tau=n / p$ ). We studied the behavior of LDSC heritability estimator for different $\omega$ and $\tau$ (Figure 1). In the first scenario, we fixed $\tau=0.1$ and varied $\omega$ from 0.005 to 1 . In the second scenario, we fixed $\omega=0.05$ and varied $\tau$ from 0.05 to 0.5 . To avoid no causal SNPs being generated when the expected causal SNP proportion $\omega$ was small, we set $(\omega / 2) p$ as a lower bound for $m$. Genotype data, SNP effect sizes and error terms were generated independently. We then used LDSC, in which all SNPs were implicitly assumed to be the causal ones, to estimate the heritability of the phenotype. The process was repeated 100 times for each setting of $\omega$ and $\tau$. As shown in Figure 1, there is almost no bias in the estimated $h^{2}$ regardless of the sample size or the underlying true model. This suggests that LDSC works well in providing an unbiased estimator of the heritability, despite the model misspecification.

Genetic covariance. We also conducted simulations on genetic covariance estimation (Figure 2). We fixed $p=20,000, \rho_{\mathrm{g}}=0.15$ and $\rho_{\mathrm{e}}=0.1$. We assumed that study 1 and study 2 are performed on the 
1.2 Numerical illustrations

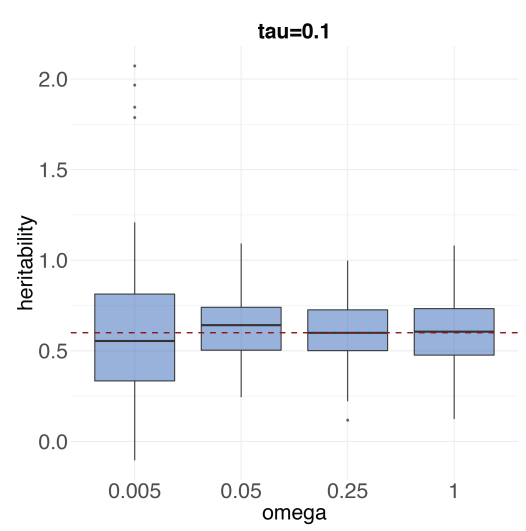

(a)

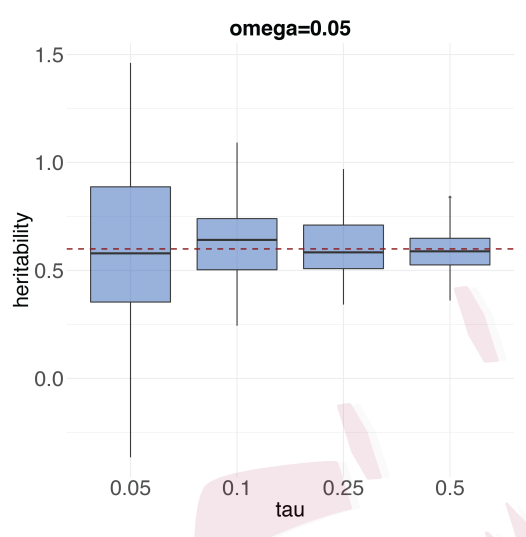

(b)

Figure 1: Heritability Estimation: $C$-dependent SNPs for $p=20,000$; (a) $\tau=0.1(n=2,000)$, and different $\omega ;$ (b) $\omega=0.05$, and different $\tau$.

same cohort. That means $n_{1}=n_{2}=n_{\mathrm{o}}$ and $X=Y$. We define that $\tau=n_{1} / p=n_{2} / p$. We used Bernoulli random variables $b_{1 j}$ and $b_{2 j}$ to indicate whether SNP $j$ is a causal SNP for phenotypes 1 and 2, respectively, such that $\mathrm{P}\left(b_{1 j}=1\right)=\omega_{1}, \mathrm{P}\left(b_{2 j}=1\right)=\omega_{2}$, and $\mathrm{P}\left(b_{1 j} b_{2 j}=1\right)=\omega$. We have $m=\sum_{j=1}^{p} b_{1 j} b_{2 j}, m+m_{1}=\sum_{j=1}^{p} b_{1 j}$, and $m+m_{2}=\sum_{j=1}^{p} b_{2 j}$. As an illustration, we assumed the causal SNPs for the two phenotypes are the same set, that is, $\omega_{1}=\omega_{2}=\omega$ and $m_{1}=m_{2}=0$. However, the consistency from this illustrative experiment can also be observed when $\omega_{1}, \omega_{2}$ and $\omega$ are not equal. Similarly, we used $(\omega / 2) p$ as a lower bound for $m$ to avoid no causal SNPs being generated. After independently generating genotype data, SNP effect sizes and error terms, we used LDSC to estimate the ge- 


\subsection{Numerical illustrations}

netic covariance of the phenotypes. All SNPs were misspecified to be the causal ones during LDSC estimation. The process was repeated 100 times for each setting of $\omega$ and $\tau$. We first fixed $\tau=0.1$ and varied $\omega$ from 0.005 to 1 . We then fixed $\omega=0.05$ and varied $\tau$ from 0.05 to 0.5 . It can be seen from Figure 2 that the LDSC estimator for genetic covariance remains unbiased under the misspecified models and different scenarios and sample sizes.

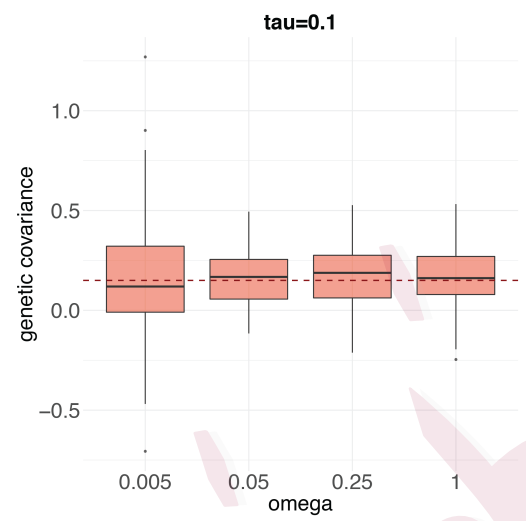

(a)

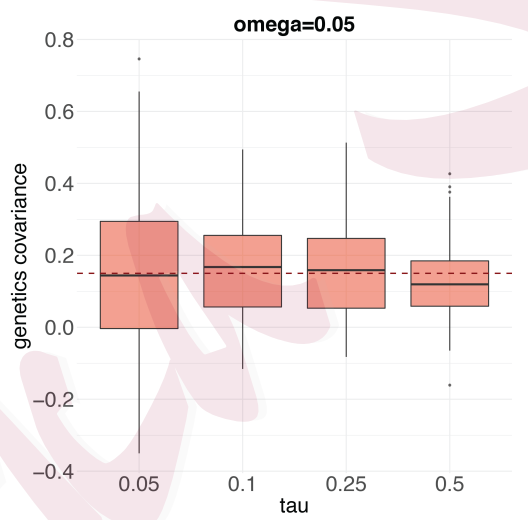

(b)

Figure 2: Genetic Covariance Estimation: $C$-dependent SNPs for $p=$ 20,000; (a) $\tau=0.1(n=2,000), \omega_{1}=\omega_{2}=0$, and different $\omega ;$ (b) $\omega_{1}=\omega_{2}=0, \omega=0.05$, and different $\tau$. 


\section{Asymptotic theory}

As noted in the previous section, there are two main quantities of genetic interest, heritability and genetic covariance. We first study the asymptotic behavior of the LDSC heritability estimator under a suitable framework. Later, we extend the framework to study the asymptotic behavior of the LDSC genetic covariance estimator. We begin with some preparation.

\subsection{Definition, key lemmas and corollary}

For any two subsets of indexes $\mathcal{B}_{r} \subset\{1, \ldots, p\}, r=1,2$, the distance between $\mathcal{B}_{1}$ and $\mathcal{B}_{2}$ is defined as

$$
d\left(\mathcal{B}_{1}, \mathcal{B}_{2}\right)=\min _{j_{1} \in \mathcal{B}_{1}, j_{2} \in \mathcal{B}_{2}}\left|j_{1}-j_{2}\right|
$$

Definition 2.1. The columns of $X$, denoted by $X_{1}, \ldots, X_{p}$, are said to be $C$-dependent, where $C$ is a constant, which may not be known, if for any subsets of $\{1, \ldots, p\}, J_{1}, \ldots, J_{t}$, such that $d\left(J_{r}, J_{s}\right)>C, 1 \leq r \neq s \leq t$, $\left[X_{j}\right]_{j \in J_{1}}, \ldots,\left[X_{j}\right]_{j \in J_{t}}$ are independent.

A standard example of $C$-dependency is the moving average process in time series (e.g., Shumway and Stoffer (2017)). There are other natural examples of $C$-dependency. Of course, one special case is the case of independent SNPs, which corresponds to $C=0$; in other words, $X_{1}, \ldots, X_{p}$ are 
independent. In practice, even if there may not be such a "cut-off", $C$, but if the correlation decays reasonably fast as the distance between the SNPs increases, after a certain distance the correlation may be treated approximately as zero. Therefore, the $C$-dependent notion is not unreasonable from a practical standpoint.It will be shown that as long as $C$ is $O(1)$, the asymptotic results do not depend on the actual value of $C$.

The technical lemmas and their corollaries are given in the Supplementary Material.

\section{$2.2 \quad$ Heritability}

We assume that the locations of the causal SNPs are characterized by a set of independent Bernoulli random variables, $b_{1}, \ldots, b_{p}$, such that $\mathrm{P}\left(b_{j}=\right.$ 1) $=\omega \in(0,1]$. Let $S=\left\{1 \leq j \leq p: b_{j}=1\right\}, m=|S|$ (cardinality), $X_{S}=\left[X_{j}\right]_{j \in S}$, and $\beta_{S}=\left(\beta_{j}\right)_{j \in S}$. It should be noted that there is a nonzero probability that $m=0$, in which case some of the quantities introduced below involving $m$ in the denominators are not well defined. However, we can (slightly) modify the definition of $m$ as $m^{*}=m \vee\{(\omega / 2) p\}$. This will not affect the kind of asymptotic behaviors studied in this paper, which are consistency or asymptotic normality. For example, let $\zeta_{N}$ denote a random variable involving $m$, and $\tilde{\zeta}_{N}$ the same quantity with $m$ replaced by $m^{*}$. 
Then, we have, for any Borel set $B$,

$$
\begin{aligned}
\mathrm{P}\left(\zeta_{N} \in B\right)= & \mathrm{P}\left[\zeta_{N} \in B, m \geq(\omega / 2) p\right]+\mathrm{P}\left[\zeta_{N} \in B, m<(\omega / 2) p\right] \\
= & \mathrm{P}\left[\tilde{\zeta}_{N} \in B, m \geq(\omega / 2) p\right]+\mathrm{P}\left[\zeta_{N} \in B, m<(\omega / 2) p\right] \\
= & \mathrm{P}\left(\tilde{\zeta}_{N} \in B\right)-\mathrm{P}\left[\tilde{\zeta}_{N} \in B, m<(\omega / 2) p\right] \\
& +\mathrm{P}\left[\zeta_{N} \in B, m<(\omega / 2) p\right] \\
= & \mathrm{P}\left(\tilde{\zeta}_{N} \in B\right)+o(1) .
\end{aligned}
$$

It can be shown that $\mathrm{P}\left(m^{*} \neq m\right)=o\left(n^{-K}\right)$ for any positive integer $K$.

Therefore, without loss of of generality, we can replace $m$ by $m^{*}$; however, for notation simplicity, we still denote it by $m$. We assume that the following hold:

(i) $X$ and $b=\left(b_{j}\right)_{1 \leq j \leq p}$ are independent;

(ii) $(X, b, \beta)$ is independent with $\epsilon$;

(iii) $\beta_{S} \mid X, b \sim N\left[0,\left(h^{2} / m\right) I_{m}\right]$, and

(iv) $\epsilon \sim N\left[0,\left(1-h^{2}\right) I_{n}\right]$.

The true underlying model can be expressed as

$$
\phi=X_{S} \beta_{S}+\epsilon=\sum_{j \in S} \beta_{j} X_{j}+\epsilon .
$$


Let $W=(X, b)$. For any $n$-dimensional constant $\lambda$, we have

$$
\begin{aligned}
\mathrm{E}\left(e^{\lambda^{\prime} \phi} \mid W\right) & =\mathrm{E}\left\{e^{\lambda^{\prime} X_{S} \beta_{S}} \mathrm{E}\left(e^{\lambda^{\prime} \epsilon} \mid W, \beta\right) \mid W\right\} \\
& =e^{\left(1-h^{2}\right) \lambda^{\prime} \lambda / 2} \mathrm{E}\left(e^{\lambda^{\prime} X_{S} \beta_{S}} \mid W\right) \\
& =e^{\lambda^{\prime} \Sigma \lambda / 2},
\end{aligned}
$$

where $\Sigma=\left(1-h^{2}\right) I_{n}+\left(h^{2} / m\right) X_{S} X_{S}^{\prime}$. It follows that $\phi \mid W \sim N(0, \Sigma)$.

It can be shown that the heritability estimator of LDSC, $\hat{h}^{2}$, can be expressed as

$$
\hat{h}^{2}=\frac{\sum_{j=1}^{p} u_{j}\left(z_{j}^{2}-1\right)}{\sum_{j=1}^{p} u_{j}^{2}},
$$

where $u_{j}=(n / p) l_{j}$. Furthermore, we have $z_{j}^{2}=\phi^{\prime}\left(n^{-1} X_{j} X_{j}^{\prime}\right) \phi$. Thus, we have

$$
\hat{h}^{2}=\phi^{\prime} A \phi-\frac{u}{u^{2}}
$$

where $u .=\sum_{j=1}^{p} u_{j}, u_{\cdot}^{2}=\sum_{j=1}^{p} u_{j}^{2}$, and $A=\left(n u_{.}^{2}\right)^{-1} \sum_{j=1}^{p} u_{j} X_{j} X_{j}^{\prime}$.

Consistency of the heritability estimator is established by the following theorem. The proof is given in the Supplementary Material.

Theorem 1. Suppose that $X_{1}, \cdots, X_{p}$ are $C$-dependent, and $n / p \longrightarrow$ $\tau \in(0,1]$. Then, we have $\hat{h}^{2}=h^{2}+o_{\mathrm{P}}(1)$.

We now consider asymptotic normality of the heritability estimator. First define

$$
r_{j_{1} j_{2} j_{3}}=\mathrm{E}\left(X_{1 j_{1}} X_{1 j_{2}}^{2} X_{1 j_{3}}\right), r_{1, j_{1} j_{2} j_{3} j_{4}}=\mathrm{E}\left(X_{1 j_{1}} X_{1 j_{2}} X_{1 j_{3}}^{2} X_{1 j_{4}}^{2}\right)
$$




$$
\begin{array}{r}
r_{2, j_{1} j_{2} j_{3}}=\mathrm{E}\left(X_{1 j_{1}}^{2} X_{1 j_{2}}^{2} X_{1 j_{3}}^{2}\right), \quad r_{2, j_{1} j_{2} j_{3} j_{4}}=\mathrm{E}\left(X_{1 j_{1}}^{2} X_{1 j_{2}}^{2} X_{1 j_{3}}^{2} X_{1 j_{4}}^{2}\right) ; \\
v_{t}=\sum_{j=1}^{p} u_{j} r_{t j}^{2}, 1 \leq t \leq p, v_{.}^{2}=\sum_{t=1}^{p} v_{t}^{2} \text {, and }(u v) .=\sum_{t=1}^{p} u_{t} v_{t} .
\end{array}
$$

Theorem 2. Suppose that, in addition to the conditions of Theorem 1 , the limits below exist: $\tau_{2}=\lim \left(u_{.}^{2} / p\right)>0, \lambda=\lim \left(u \cdot / u_{.}^{2}\right), \phi=$ $\lim \left\{(u v) . / u^{2}\right\}$

$$
\begin{aligned}
& \gamma=\lim \left\{\left(u_{.}^{2}\right)^{2} / n v_{.}^{2}\right\}>0 \\
& \gamma_{1}=\lim p^{-1} \sum_{j_{1}, j_{2}, j_{3}, j_{4}=1}^{p} u_{j_{1}} u_{j_{3}} r_{j_{1} j_{2}} r_{j_{2} j_{3}} r_{j_{3} j_{4}} r_{j_{4} j_{1}}, \\
& \gamma_{2}=\lim p^{-2} \sum_{j_{1}, j_{2}, j_{3}, j_{4}=1}^{p} u_{j_{1}} u_{j_{3}} r_{j_{1} j_{2} j_{3}} r_{j_{3} j_{4}} r_{j_{4} j_{1}}, \\
& \gamma_{3}=\lim p^{-3} \sum_{j_{1}, j_{2}, j_{3}, j_{4}=1}^{p} u_{j_{1}} u_{j_{3}} r_{j_{1} j_{2}} r_{1, j_{1} j_{2} j_{3} j_{4}}, \\
& \gamma_{4}=\lim p^{-3} \sum_{j_{1}, j_{2}, j_{3}, j_{4}=1}^{p} u_{j_{1}} u_{j_{3}} r_{j_{1} j_{2} j_{3}} r_{j_{3} j_{4} j_{1}} \\
& \gamma_{5}=\lim p^{-4} \sum_{j_{1}, j_{2}, j_{3}, j_{4}=1}^{p} u_{j_{1}} u_{j_{3}} r_{2, j_{1} j_{2} j_{3} j_{4}}, \\
& \gamma_{6}=\lim p^{-1} \sum_{j_{1}, j_{2}, j_{3}=1}^{p} u_{j_{1}} u_{j_{2}} r_{j_{1} j_{2}} r_{j_{2} j_{3} r_{j_{3}} r_{1}}, \\
& \gamma_{7}=\lim p^{-2} \sum_{j_{1}, j_{2}, j_{3}=1}^{p} u_{j_{1}} u_{j_{2}}\left(r_{j_{1} j_{2}} r_{j_{2} j_{3} j_{1}}+r_{j_{2} j_{3}} r_{j_{3} j_{1} j_{2}}+r_{j_{3} j_{1}} r_{j_{1} j_{2} j_{3}}\right)
\end{aligned}
$$$$
\text { and } \gamma_{8}=\lim p^{-3} \sum_{j_{1}, j_{2}, j_{3}=1}^{p} u_{j_{1}} u_{j_{3}} r_{2, j_{1} j_{2} j_{3}} \text {, as } p \rightarrow \infty \text {. Then, we have }
$$

$$
\sqrt{n}\left(\hat{h}^{2}-h^{2}\right) \stackrel{\mathrm{d}}{\longrightarrow} N\left(0, \sigma^{2}\right)
$$

as $p \rightarrow \infty$, where the asymptotic variance has the following expression:

$$
\begin{aligned}
& \sigma^{2}=3 h^{4} \tau\left(\frac{1-\omega}{\omega}\right)\left(\frac{\tau}{\gamma}-1\right) \\
& +2\left[h^{4}\left\{\frac{\gamma_{1} \tau^{3}+6 \gamma_{2} \tau^{2}+\left(4 \gamma_{3}+2 \gamma_{4}\right) \tau+\gamma_{5}}{\tau_{2}^{2}}+\left(\frac{1-\omega}{\omega}\right) \tau(\lambda+1)^{2}\right\}\right. \\
& \left.+2 h^{2}\left(1-h^{2}\right) \frac{\gamma_{6} \tau^{2}+\gamma_{7} \tau+\gamma_{8}}{\tau_{2}^{2}}+\left(1-h^{2}\right)^{2}\left(\frac{\phi \tau}{\tau_{2}}+\lambda^{2}\right)\right]
\end{aligned}
$$




\subsection{Heritability}

Inspection of the limits defined in Theorem 2, in terms of the order of the sum involved in the limit, suggests that the limits can all be reasonably expected. The proof of Theorem 2 is given in the Supplementary Material.

A special case is when the SNPs are independent, that is, $C=0$. In this case, it can be verified that $\tau_{2}=\tau^{2}, \lambda=\tau^{-1}, \gamma=\tau, \phi=1, \gamma_{s}=\tau^{2}$, if $1 \leq s \leq 8$ and $s \neq 7$, and $\gamma_{7}=3 \tau^{2}$, where $\tau$ is given by Theorem 2. Thus, we have the following result.

Corollary 2. In the case of independent SNPs, 2.10 holds under $n / p \longrightarrow \tau \in(0,1]$, where

$$
\begin{aligned}
& \sigma^{2} \equiv 2\left[\frac{1}{\tau}+h^{4}\left\{\frac{\tau}{\omega}\left(1+\frac{1}{\tau}\right)^{2}+\left(2+\frac{1}{\tau}\right)^{2}\right\}\right. \\
& \left.+2 h^{2}\left(1-h^{2}\right)\left(1+\frac{1}{\tau}\right)^{2}+\frac{\left(1-h^{2}\right)^{2}}{\tau^{2}}\right] .
\end{aligned}
$$

We note that since $\omega, \tau \in(0,1]$, we have $\sigma^{2}=O(1)$ and hence the convergence rate of $\hat{h}^{2}$ is $n^{-1 / 2}$. Because $m$ and $n$ are of the same order, the convergence rate can be expressed either in terms of $n$ or in terms of $m$. In fact, as the asymptotic depend on both $\omega$ and $\tau$, the asymptotic variance depends on the (limit) ratio of $m / n$, which makes sense. 


\subsection{Genetic covariance}

Let $b_{r j}, 1 \leq j \leq p, r=1,2$ be such that

(I) $\left(b_{1 j}, b_{2 j}\right), j=1, \ldots, p$ are independent;

(II) $b_{r j} \sim \operatorname{Bernoulli}\left(\omega_{r}\right), j=1,2$, where $\omega_{1}, \omega_{2} \in(0,1]$;

(III) $\omega=\mathrm{P}\left(b_{1 j} b_{2 j}=1\right)>0$.

Note that the definition allows correlation between $b_{1 j}$ and $b_{2 j}$ for the same

$j$. Note that $\omega=\omega_{1} \omega_{2}$ if $b_{1 j}, b_{2 j}$ are independent. Denote $b_{r}=\left(b_{r j}\right)_{1 \leq j \leq p}$, $r=1,2$, and $b=\left(b_{1}, b_{2}\right)$. Then, we have $S=\left\{1 \leq j \leq p: b_{1 j} b_{2 j}=1\right\}$, $m=|S| ; S \cup T_{r}=\left\{1 \leq j \leq p: b_{r j}=1\right\}, m_{r}=\left|S \cup T_{r}\right|-|S|=\left|S \cup T_{r}\right|-m$, so $\left|S \cup T_{r}\right|=m+m_{r}, r=1,2$. For any subset of indexes $J \subset\{1, \ldots, p\}$, let $X_{J}=\left[X_{j}\right]_{j \in J}$ and $Y_{J}$ is defined similarly. Let $\beta=\left(\beta_{S}^{\prime}, \beta_{T_{1}}^{\prime}, \beta_{T_{2}}^{\prime}, \beta_{U}^{\prime}\right)^{\prime}$, $\gamma=\left(\gamma_{S}^{\prime}, \gamma_{T_{1}}^{\prime}, \gamma_{T_{2}}^{\prime}, \gamma_{U}^{\prime}\right)^{\prime}$. We assume that the following conditions hold:

(a) $(X, Y)$ and $b$ are independent;

(b) $(X, Y, \beta, \gamma, b)$ is independent with $(\epsilon, \delta)$;

(c) $(\beta, \gamma) \mid b \sim N(0, \Omega)$, where $\Omega$ is the covariance matrix described in Section 1

(d) $(\epsilon, \delta) \sim$ the distribution specified as in Section 1 .

It is more convenient to define $\beta_{j}=0, j \notin S \cup T_{1}$ and $\gamma_{j}=0, j \notin S \cup T_{2}$. Let $\xi_{j}=\left(\xi_{1 j}, \xi_{2 j}\right)^{\prime}=\left(\sqrt{m+m_{1}} b_{1 j} \beta_{j}, \sqrt{m+m_{2}} b_{2 j} \gamma_{j}\right)^{\prime}, 1 \leq j \leq p$. Then, 


\subsection{Genetic covariance}

given $b, \xi_{j}, 1 \leq j \leq p$ are independent vectors with $\xi_{j} \sim N\left(0, \Sigma_{b}\right)$, where

$$
\Sigma_{b}=\left[\begin{array}{cc}
h_{1}^{2} & \rho_{\mathrm{g}} \sqrt{\left(1+m_{1} / m\right)\left(1+m_{2} / m\right)} \\
\rho_{\mathrm{g}} \sqrt{\left(1+m_{1} / m\right)\left(1+m_{2} / m\right)} & h_{2}^{2}
\end{array}\right],
$$

$j \in S, \xi_{j}=\left(\xi_{1 j}, 0\right)^{\prime}$ with $\xi_{1 j} \sim N\left(0, h_{1}^{2}\right), j \in T_{1} ; \xi_{j}=\left(0, \xi_{2 j}\right)^{\prime}$ with $\xi_{2 j} \sim$ $N\left(0, h_{2}^{2}\right), j \in T_{2} ;$ and $\xi_{j}=0, j \notin S \cup T_{1} \cup T_{2}$. Let $\xi_{b}=\left(\xi_{j}\right)_{1 \leq j \leq p}$, the column vector that combines all of the $\xi_{j}$ s. It should be noted that, for $\beta_{S}, \gamma_{S}$ to have the joint distribution as specified in Section 1, it is necessary that the following holds:

$$
\frac{\rho_{\mathrm{g}}}{m}=\left|\operatorname{cov}\left(\beta_{j}, \gamma_{j}\right)\right| \leq \sqrt{\operatorname{var}\left(\beta_{j}\right) \operatorname{var}\left(\gamma_{j}\right)}=\frac{h_{1} h_{2}}{\sqrt{\left(m+m_{1}\right)\left(m+m_{2}\right)}},
$$

$j \in S$, if $S \neq \emptyset$. It follows that the following inequality must be satisfied:

$$
\left(1+\frac{m_{1}}{m}\right)\left(1+\frac{m_{2}}{m}\right) \leq\left(\frac{h_{1} h_{2}}{\rho_{\mathrm{g}}}\right)^{2} .
$$

Therefore, we modify the definition of the covariance matrix of $\beta$ and $\gamma$ so that, when (2.13) does not hold, the covariance matrix of $\beta_{S}$ and $\gamma_{S}$ is 0 (matrix). As a result, the covariance matrix of $\xi_{j}$ is $\operatorname{diag}\left(h_{1}^{2}, h_{2}^{2}\right), 1 \leq j \leq p$ when (2.13) does not hold. It can be seen that 2.13) holds with probability tending to one provided that

$$
\frac{\omega_{1} \omega_{2}}{\omega^{2}}<\left(\frac{h_{1} h_{2}}{\rho_{\mathrm{g}}}\right)^{2}
$$

Therefore, asymptotically, $\left(\beta_{S}, \gamma_{S}\right)$ still has the distribution described in Section 1. 


\subsection{Genetic covariance}

The LDSC estimator of the genetic covariance is defined differently under independent SNPs than under correlated SNPs. We consider these cases separately.

1. Independent SNPs. In this case, we assume $n_{\mathrm{o}}=0$ to ensure identifiability. Then, the LDSC estimator of $\rho_{\mathrm{g}}$ is simplified to

$$
\hat{\rho}_{\mathrm{g}}=\frac{1}{n_{1} n_{2}} \sum_{j=1}^{p} \phi_{1}^{\prime} X_{j} Y_{j}^{\prime} \phi_{2}=\phi^{\prime} A \phi,
$$

where $\phi=\left(\phi_{1}^{\prime}, \phi_{2}^{\prime}\right)^{\prime}$ and $A=\left(2 n_{1} n_{2}\right)^{-1} \sum_{j=1}^{p} \Psi_{j}$ with

$$
\Psi_{j}=\left(\begin{array}{cc}
0 & X_{j} Y_{j}^{\prime} \\
Y_{j} X_{j}^{\prime} & 0
\end{array}\right) .
$$

The following result is proved in the Supplementary Material.

Theorem 3. Suppose that the SNPs are independent, 2.14 holds with $\omega>0$, and

$$
\frac{n_{r}}{p} \rightarrow \tau_{r} \in(0,1], r=1,2 .
$$

Then, we have $\hat{\rho}_{\mathrm{g}}=\rho_{\mathrm{g}}+o_{\mathrm{P}}(1)$.

The next result is regarding asymptotic distribution of $\hat{\rho}_{\mathrm{g}}$. The proof is given in the Supplementary Material.

Theorem 4. Under the conditions of Theorem 1, we have $\sqrt{n}$. $\left(\hat{\rho}_{\mathrm{g}}-\right.$ 
$\left.\rho_{\mathrm{g}}\right) \stackrel{\mathrm{d}}{\longrightarrow} N\left(0, \sigma^{2}\right)$, where $n .=n_{1}+n_{2}$, and

$$
\begin{aligned}
\sigma^{2}= & \left(\tau_{1}+\tau_{2}\right)\left\{h_{1}^{2} h_{2}^{2}\left(\frac{\omega}{\omega_{1} \omega_{2}}+\frac{\tau_{1}+\tau_{2}+1}{\tau_{1} \tau_{2}}\right)+\rho_{\mathrm{g}}^{2}\left(\frac{1}{\omega}+\frac{1}{\tau_{1}}+\frac{1}{\tau_{2}}\right)\right\} \\
& +\left(\frac{1}{\tau_{1}}+\frac{1}{\tau_{2}}\right)\left\{h_{1}^{2}\left(1-h_{2}^{2}\right) \tau_{1}+\left(1-h_{1}^{2}\right) h_{2}^{2} \tau_{2}+1-h_{1}^{2} h_{2}^{2}\right\} .
\end{aligned}
$$

2. C-dependent SNPs. In this case, the genetic covariance, $\rho_{\mathrm{g}}$, is estimated by fitting the following linear regression in LDSC

$$
z_{j}=\beta_{0}+\beta_{1} u_{j}+e_{j}, \quad j=1, \ldots, p
$$

where $z_{j}=z_{1 j} z_{2 j}, u_{j}=\left(\sqrt{n_{1} n_{2}} / p\right) l_{j}$ and $\beta_{1}=\rho_{\mathrm{g}}$. The LDSC estimators, which are also the least squares (LS) estimators of the regression coefficients, are given by

$$
\begin{aligned}
& \hat{\rho}_{\mathrm{g}}=\hat{\beta}_{1}=\frac{\sum_{j=1}^{p}\left(u_{j}-\bar{u}\right)\left(z_{j}-\bar{z}\right)}{\sum_{j=1}^{p}\left(u_{j}-\bar{u}\right)^{2}}=\phi^{\prime} A \phi, \\
& \hat{\beta}_{0}=\bar{z}-\hat{\beta}_{1} \bar{u}
\end{aligned}
$$

where $A=\left(2 \sqrt{n_{1} n_{2}} d_{p}\right)^{-1} \sum_{j=1}^{p}\left(u_{j}-\bar{u}\right) \Psi_{j}, d_{p}=\sum_{j=1}^{p}\left(u_{j}-\bar{u}\right)^{2}, \Psi_{j}$ given by 2.16), $\bar{u}=p^{-1} \sum_{k=1}^{p} u_{k}$, and $\bar{z}=p^{-1} \sum_{k=1}^{p} z_{k}$. As our main interest is estimating $\rho_{\mathrm{g}}$, we shall focus on $\hat{\beta}_{1}=\hat{\rho}_{\mathrm{g}}$. Theorem 5 below states consistency of the estimator.

Theorem 5. Suppose that the SNPs are $C$-dependent, $\omega>0$, 2.14, 2.17 hold, and $d_{p} / \sqrt{p} \rightarrow \infty$. Then, we have $\hat{\rho}_{\mathrm{g}}=\hat{\beta}_{1}=\rho_{\mathrm{g}}+o_{\mathrm{P}}(1)$. 


\subsection{Genetic covariance}

Note that, under 2.17$), d_{p} / \sqrt{p} \rightarrow \infty$ iff $\sum_{j=1}^{p}\left(l_{j}-\bar{l}\right)^{2} / \sqrt{p} \rightarrow \infty$, where $l_{j}$ is the LD score and $\bar{l}=p^{-1} \sum_{j=1}^{p} l_{j}$. The proof of Theorem 5 is given in the Supplementary Material.

Next we consider asymptotic distribution of $\hat{\rho}_{\mathrm{g}}$. The result is relatively simpler in terms of the asymptotic variance under the assumption that

$$
n_{\mathrm{o}}=o\left(n_{1} \wedge n_{2}\right)
$$

Thus, we consider this special case first. Define the following quantities:

$\rho_{b}=\operatorname{cov}\left(b_{1 j}, b_{2 j}\right)=\omega-\omega_{1} \omega_{2}, \psi_{0}=d_{p} / p, \psi_{1}=p^{-1} \sum_{j, k=1}^{p}\left(u_{j}-\bar{u}\right)\left(u_{k}-\bar{u}\right) r_{j k}^{2}$,

$\psi_{2, s}=\sum_{j, k=1}^{p}\left(u_{j}-\bar{u}\right)\left(u_{k}-\bar{u}\right) r_{j k} r_{k s} r_{s j}, \psi_{3, s}=p^{-1} \sum_{j, k=1}^{p}\left(u_{j}-\bar{u}\right)\left(u_{k}-\right.$ $\bar{u}) r_{j k} r_{k s j} ; \psi_{1}(s, t)=\mathrm{E}\left(h_{1, s, t}^{2}\right)$ and $\psi_{2}(s, t)=\mathrm{E}\left(h_{1, s, t} h_{2, s, t}\right)$, where $h_{1, s, t}$ and $h_{2, s, t}$ are the $(s, t)$ elements of $H_{1}=X^{\prime} X D Y^{\prime} Y$ and $H_{2}=Y^{\prime} Y D X^{\prime} X$, respectively, where $D=\operatorname{diag}\left(u_{j}-\bar{u}, 1 \leq j \leq p\right)$.

Theorem 6. Suppose that the SNPs are $C$-dependent, $\omega>0$, 2.14, 2.17), and 2.22 hold. Also suppose that the following identities hold for all $j, k, s$ :

$$
\mathrm{E}\left(X_{1 j} X_{1 k}^{2} X_{1 s}\right)=\mathrm{E}\left(Y_{1 j} Y_{1 k}^{2} Y_{1 s}\right), \mathrm{E}\left(X_{1 j}^{2} X_{1 k}^{2} X_{1 s}^{2}\right)=\mathrm{E}\left(Y_{1 j}^{2} Y_{1 k}^{2} Y_{1 s}^{2}\right)
$$

Furthermore, suppose that the following limits exist as $p \rightarrow \infty$ : $\phi_{0}=$ $\lim \psi_{0}>0, \phi_{1}=\lim \psi_{1}, \phi_{r}=\lim \left(n . / p^{2}\right) \sum_{s=1}^{p} \psi_{r, s}, r=2,3$, and

$$
\vartheta_{1}=\lim \frac{n \cdot n_{1} n_{2}}{p^{4}} \sum_{t=1}^{p}\left\{\sum_{s=1}^{p}\left(u_{s}-\bar{u}\right) r_{s t}^{2}\right\}^{2},
$$




$$
\lambda_{r}=\lim \frac{n .}{n_{1} n_{2} p^{4}} \sum_{s, t=1}^{p} \psi_{r}(s, t), \quad \mu_{r}=\lim \frac{n .}{n_{1} n_{2} p^{4}} \sum_{s=1}^{p} \psi_{r}(s, s),
$$

$r=1,2$. Then, we have $\sqrt{n} .\left(\hat{\rho}_{\mathrm{g}}-\rho_{\mathrm{g}}\right) \stackrel{\mathrm{d}}{\longrightarrow} N\left(0, \sigma^{2}\right)$, where $\sigma^{2}=\sigma_{1}^{2}+\sigma_{2}^{2}$ with

$$
\begin{aligned}
& \sigma_{1}^{2}=\rho_{\mathrm{g}}^{2}\left(\frac{1}{\omega}-1\right)\left(\frac{\vartheta_{1}}{\phi_{0}^{2}}-\tau_{1}-\tau_{2}\right) \\
& \sigma_{2}^{2}=\frac{1}{\phi_{0}^{2}}\left\{h_{1}^{2} h_{2}^{2}\left(\lambda_{1}-\mu_{1}+\frac{\omega \mu_{1}}{\omega_{1} \omega_{2}}\right)+h_{1}^{2}\left(1-h_{2}^{2}\right)\left(\tau_{1} \phi_{2}+\phi_{3}\right)\right. \\
& +\left(1-h_{1}^{2}\right) h_{2}^{2}\left(\tau_{2} \phi_{2}+\phi_{3}\right)+\left(1-h_{1}^{2}\right)\left(1-h_{2}^{2}\right)\left(\tau_{1}+\tau_{2}\right) \phi_{1} \\
& \left.+\rho_{\mathrm{g}}^{2}\left(\lambda_{2}-\mu_{2}+\frac{\mu_{2}}{\omega}\right)\right\}
\end{aligned}
$$

It is seen that, in the case that $b_{1 j}, b_{2 j}$ are uncorrelated, that is $\omega=\omega_{1} \omega_{2}$, the above asymptotic variance, $\sigma^{2}$, depends on $\omega^{-1}$ but not on $\omega_{1}$ and $\omega_{2}$. A similar observation is made regarding (2.18). The proof of Theorem 6 is given in the Supplementary Material.

Finally, we extend Theorem 6 without requiring 2.22 . First define the following additional quantities: $\psi_{r}(i, t)=\mathrm{E}\left(h_{r, i, t}^{2}\right), r=3,4$, where $h_{3, i, t}, h_{4, i, t}$ are the $(i, t)$ elements of $H_{3}=X D Y^{\prime} Y, H_{4}=Y D X^{\prime} X$, respectively, and $\psi_{5}(i, t)=\mathrm{E}\left(h_{3, i, t} h_{4, i, t}\right)$. Also define $\psi_{6}\left(i_{1}, i_{2}\right)=\mathrm{E}\left(h_{5, i_{1}, i_{2}}^{2}\right)$, where $h_{5, i_{1}, i_{2}}$ is the $\left(i_{1}, i_{2}\right)$ element of $H_{5}=X D Y^{\prime}$. We now have the extension of Theorem 6.

Theorem 7. Suppose that the conditions of Theorem 6 without 2.22) 
hold. In addition, suppose that the following limits exist:

$$
\begin{aligned}
& \lambda_{r}=\lim \frac{n .}{n_{1} n_{2} p^{3}} \sum_{i=1}^{n_{r-2}} \sum_{t=1}^{p} \psi_{r}(i, t), \quad r=3,4, \\
& \lambda_{5, \mathrm{o}}=\lim \frac{n .}{n_{1} n_{2} p^{3}} \sum_{i=1}^{n_{\mathrm{o}}} \sum_{t=1}^{p} \psi_{5}(i, t), \\
& \lambda_{6}=\frac{n .}{n_{1} n_{2} p^{2}} \sum_{i_{1}=1}^{n_{1}} \sum_{i_{2}=1}^{n_{2}} \psi_{6}\left(i_{1}, i_{2}\right), \lambda_{6, \mathrm{o}}=\frac{n .}{n_{1} n_{2} p^{2}} \sum_{i_{1}, i_{2}=1}^{n_{\mathrm{o}}} \psi_{6}\left(i_{1}, i_{2}\right) .
\end{aligned}
$$

Then, the conclusion of Theorem 6 holds with $\sigma_{2}^{2}$ replaced by the following:

$$
\begin{aligned}
\sigma_{2}^{2}= & \frac{1}{\phi_{0}^{2}}\left[h_{1}^{2} h_{2}^{2}\left(\lambda_{1}-\mu_{1}+\frac{\omega \mu_{1}}{\omega_{1} \omega_{2}}\right)+\rho_{\mathrm{g}}^{2}\left(\lambda_{2}-\mu_{2}+\frac{\mu_{2}}{\omega_{2}}\right)\right. \\
& +\left(1-h_{1}^{2}\right) h_{2}^{2} \lambda_{3}+h_{1}^{2}\left(1-h_{2}^{2}\right) \lambda_{4}+2 \rho_{\mathrm{e}} \rho_{\mathrm{g}} \lambda_{5, \mathrm{o}} \\
& \left.+\left(1-h_{1}^{2}\right)\left(1-h_{2}^{2}\right) \lambda_{6}+\left\{\rho_{\mathrm{e}}+\frac{\left(h_{1}^{2}-h_{2}^{2}\right)^{2}}{2}\right\} \lambda_{6, \mathrm{o}}\right] .
\end{aligned}
$$

The proof of Theorem 7 is given in the Supplementary Material.

\section{Simulation studies}

We carried out comprehensive simulation to numerically validate our theoretical results. In these experiments, we evaluated the consistency of the summary-statistics-based heritability and genetic covariance estimators under model misspecification. Besides, we compared the empirical distributions of these estimators with the asymptotic distributions derived from our theory. Unless explicitly stated, the heritability and genetic covariance estimators refer to those described in the previous sections. 


\subsection{Heritability}

\subsection{Heritability}

It is shown in Figure 1.2 that the heritability estimator is nearly unbiased even under model misspecification. Following the same settings introduced in Section 1.2, we computed observed standard deviation of the heritability estimator during 100 runs for each combination of underlying model parameters (namely $\tau$ and $\omega$ ). Then we also computed the corresponding theoretical standard errors of the estimators under different model settings by using the formula derived in Theorem 2. All the limits presented in Theorem 2 are computed based on their corresponding observed values. For example, $\tau_{2}$ is replaced by $u_{.}^{2} / p$. As is shown in Table 3.1, the values of standard errors derived from our theory are very close to the observed standard errors under different combinations of $\tau$ and $\omega$. Further evaluation results of consistency and asymptotic normality of the heritability estimator are included in the Supplementary Material. The consistency and asymptotic normality of the heritability estimators under different settings looks good.

\subsection{Genetic covariance}

In the numerical illustrations presented in Section 1.2 , we demonstrated approximate unbiasedness of the genetic covariance estimator when the 
3.2 Genetic covariance

\begin{tabular}{ccc|ccc}
\hline & \multicolumn{2}{|c}{$\tau=0.1$} & \multicolumn{3}{c}{$\omega=0.05$} \\
$\omega$ & observed & theoretical & $\tau$ & observed & theoretical \\
\hline 0.005 & 0.41 & 0.39 & 0.05 & 0.37 & 0.36 \\
0.05 & 0.17 & 0.20 & 0.1 & 0.17 & 0.20 \\
0.25 & 0.17 & 0.18 & 0.25 & 0.13 & 0.13 \\
1 & 0.18 & 0.17 & 0.5 & 0.10 & 0.11 \\
\hline
\end{tabular}

Table 1: Observed and Theoretical Standard Errors of Heritability Estimator

two studies share the same set of subjects. However, in practice, there is often only a small portion or even none of the subjects shared among two GWAS, especially when they come from different cohorts. Here we set $n_{\mathrm{o}} / n=0.1$, where $n=n_{1}=n_{2}$. All other settings are the same as those described in Section 1.2. We calculated the observed standard deviation of the genetic covariance estimator based on 100 simulation runs under each parameter setting. We then computed the theoretical standard errors derived from Theorem 7. Similarly, the limits presented in the theorem are determined by their corresponding observed values. The comparisons of the observed and theoretical standard errors are shown in Table 3.2. The theoretical standard errors are close to the observed standard deviation, confirming our results of Theorem 6/Theorem 7 . We further investigated consistency and asymptotic normality of the genetic covariance estimator. The empirical results are presented in the Supplementary Material. The 


\subsection{Genetic covariance}

consistency and asymptotic normality of the genetic covariance estimator looks good under all settings. We also added additional simulations to investigate the relationship between the efficiency loss of LDSC and model sparsity in the Supplementary Material. We found that the sparser the true model is the more efficiency loss there would be. That coincides with our intuition, since LD score regression is developed based on polygenetic assumption.

\begin{tabular}{ccc|ccc}
\hline \multicolumn{3}{c|}{$\tau=0.1, n_{\mathrm{o}} / n=0.1$} & \multicolumn{3}{c}{$\omega=0.05, n_{\mathrm{o}} / n=0.1$} \\
$\omega$ & observed & theoretical & $\tau$ & observed & theoretical \\
\hline 0.005 & 0.28 & 0.26 & 0.05 & 0.29 & 0.24 \\
0.05 & 0.17 & 0.17 & 0.1 & 0.17 & 0.17 \\
0.25 & 0.17 & 0.15 & 0.25 & 0.14 & 0.12 \\
1 & 0.17 & 0.15 & 0.5 & 0.11 & 0.11 \\
\hline
\end{tabular}

Table 2: Observed and Theoretical Standard Errors of Genetic Covariance Estimator

Next, we provide a real data example that applies LDSC to estimate the heritability and genetic covariance among four lipid traits: high-density lipoprotein (HDL) cholesterol, low-density lipoprotein (LDL) cholesterol, total cholesterol (TC), and triglyceride (TG). In the example, we compare the results from LDSC with REML estimates. The results are in the supplementary materials. 


\section{Discussion}

LDSC has become the most popular method to estimate heritability and genetic correlation due to its efficiency and simplicity. In this paper, we studied the consistency and asymptotic normality of LDSC under misspecified model. While LDSC is based on random effects model, there are several method proposed to estimate heritability and genetic correlation based on fixed effects model (Shi et al. (2017), Shi, Kichaev and Pasaniuc (2016), Wang and Li (2021), Guo et al. (2019)). It has been shown that under the assumption of the random effects model, the estimator of the fixed effects model would converge to the estimator of LDSC almost surely (Wang and Li |(2021)). When the assumption does not hold, neither of the model has its advantages which can overwhelm the other. One advantage of the random effects model is that it incorporates implicit and automatic regularization of the regression coefficients, unlike in case of a sparse fixed effects model. The latter requires a careful choice of the penalty/thresholding parameters to be effective. Also, the random effects model allows for a systematic mechanism for carrying out statistical inference. This is in essence achieved through the asymptotic distribution of estimated heritability and genetic correlation. Besides, methods based on the random effects model are generally more computationally efficient. The fixed effects model involves the 
calculation of the inverse of LD matrix which needs extra assumption that LD matrix is block-diagonal. On the other hand, methods based on the fixed effects model require fewer assumptions on genetic effects. Therefore, some of the researchers thought it is more robust across a wide range of genetic architectures such as sparse causal SNPs (Wang and Li (2021)). However, in this work, we proved that LDSC can also provide consistent estimator under model misspecification.

The LDAK model (Speed and Balding (2019)) assumed that the variances of the SNP effects of the standardized SNPs are proportional to a set of known parameters $q_{1}, q_{2}, \ldots, q_{p}$, where $p$ is the number of SNPs. This model can be seen as the generalization of LDSC. Indeed, when $q_{1}=q_{2}=$ $\ldots=q_{p}, \mathrm{LDAK}$ reduces to LDSC. In practice, the value of $q_{i}$ for $\mathrm{SNP} i$ is a function of the minor allele frequency (MAF) of SNP $i, f_{i}$. Our results can be extended to LDAK model. Under the model of LDSC, we have $E\left(z_{j}^{2}\right)=1+h^{2}(n / p) l_{j}$, where $j=1,2, \ldots, p$. Instead, in LDAK, the regression problem changes to $E\left(z_{j}^{2}\right)=1+n h^{2}\left(\sum_{k=1}^{p} r_{j k}^{2} q_{k}\right) / \sum_{k=1}^{p} q_{k}$, where $r_{j k}$ is the correlation between SNP $j$ and SNP $k$. Under appropriate assumption for $q_{1}, q_{2}, \ldots, q_{p}$, the term $\left(\sum_{k=1}^{p} r_{j k}^{2} q_{k}\right) p / \sum_{k=1}^{p} q_{k}$ is interchangeable with $l_{j}$. However, for simplicity and also due to the space limit, we will leave the extension to a future work. 
There are certain limitations in our theoretical assumptions. First, in practice, since the true LD matrix is unknown to us, we have to use external reference panel to estimate LD score. If the external data source used to estimate the LD scores is of higher order than $n$, which is the sample size of the GWAS, the asymptotic results, both consistency and asymptotic normality, will not be affected. If the external sample size is the of the same order as $n$, consistency is not affected but the asymptotic distribution will change. Second, we assumed the constant $C$ in $C$-dependent assumption if of $O(1)$. Actually, it is possible to allow $C$ to increase, slowly, with $n$, so that the asymptotic results do not change. However, if the order of $C$ exceeds a certain threshold, the asymptotic distribution, even consistency result, may change.

\section{Supplementary Materials}

Proofs and additional empirical results.

\section{Acknowledgements}

The authors would like to thank the anonymous referees, an Associate Editor and the Editor for their constructive comments that improved the quality of this paper. Jiming Jiang and Debashis Paul's research is supported 


\section{REFERENCES}

by NSF grant DMS-1713120. Wei Jiang and Yiliang Zhang's research is supported by NSF grant DMS-1902903 and NIH grant R01 GM134005. Hongyu Zhao's research is supported by NSF grants DMS-1713120 and DMS-1902903 and NIH grant R01 GM134005. We conducted the research using the UKBB resource under approved data requests (access ref: 29900).

\section{References}

Berisa, T. and Pickrell, J. K. (2016), Approximately independent linkage disequilibrium blocks in human populations, Bioinformatics 32, 283.

Berndt, S. I., Gustafsson, S., Mägi, R., Ganna, A., Wheeler, E., Feitosa, M. F., Justice, A. E., Monda, K. L., Croteau-Chonka, D. C., Day, F. R. and others (2013), Genome-wide meta-analysis identifies 11 new loci for anthropometric traits and provides insights into genetic architecture, Nature Genetics 45, 501-512.

Bulik-Sullivan, B. K., Loh, P.-R., Finucane, H. K., Ripke, S., Yang, J., Patterson, N., Daly, M. J., Price, A. L., and Neale, B. M. (2015a), LD Score regression distinguishes confounding from polygenicity in genome-wide association studies, Nature Genetics 47, 291-295.

Bulik-Sullivan, B., K., Finucane, H. K., Anttila, V., Gusev, A., Day, F. R., Loh, P.-R., Duncan, L., Perry, J. R. B., Patterson, N., Robinson, E. B. and others (2015b), An atlas of genetic correlations across human diseases and traits, Nature Genetics 47, 1236.

Buniello, A., MacArthur, J. A. L., Cerezo, M., Harris, L. W., Hayhurst, J., Malangone, C., 


\section{REFERENCES}

McMahon, A., Morales, J., Mountjoy, E., Sollis, E. and others (2019), The NHGRI-EBI

GWAS Catalog of published genome-wide association studies, targeted arrays and summary statistics 2019, Nucleic Acids Research 47, D1005-D1012.

Clarke, L., Fairley, S., Zheng-Bradley, X., Streeter, I., Perry, E., Lowy, E., Tassé, A.-M., and Flicek, P. (2017), The international Genome sample resource (IGSR): A worldwide collection of genome variation incorporating the 1000 Genomes Project data, Nucleic Acids Research 45, D854-D859.

Guo, Z., Wang, W., Cai, T.T. and Li, H., (2019), Optimal estimation of genetic relatedness in high-dimensional linear models, Journal of the American Statistical Association, 114(525), pp.358-369.

Jiang, J. (2010). Large Sample Techniques for Statistics, Springer, New York.

Jiang, J., Li, C., Paul, D., Yang, C., and Zhao, H. (2016), On high-dimensional misspecified mixed model analysis in genome-wide association study, The Annals of Statistics 44, 21272160 .

Jiang, W., Song, S., Hou, L., and Zhao, H. (2020), A set of efficient methods to generate high-dimensional binary data with specified correlation structures, arXiv:2007.14080.

Lee, S. H., Yang, J., Goddard, M. E., Visscher, P. M., and Wray, N. R. (2012), Estimation of pleiotropy between complex diseases using SNP-derived genomic relationships and restricted maximum likelihood, Bioinformatics 28, 2540-2542.

Shi, H., Kichaev, G. and Pasaniuc, B., (2016), Contrasting the genetic architecture of 30 complex 


\section{REFERENCES}

traits from summary association data. The American Journal of Human Genetics, 99(1), pp.139-153.

Shi, H., Mancuso, N., Spendlove, S. and Pasaniuc, B., (2017), Local genetic correlation gives insights into the shared genetic architecture of complex traits, The American Journal of Human Genetics, 101(5), pp.737-751.

Shumway, R. H. and Stoffer, D. S. (2017), Time Series Analysis and Its Applications, Springer International Publishing AG.

Speed, D. and Balding, D.J., (2019), SumHer better estimates the SNP heritability of complex traits from summary statistics, Nature genetics, 51(2), pp.277-284.

Stephens, J. C., Schneider, J. A., Tanguay, D. A., Choi, J., Acharya, T., Stanley, S. E., Jiang, R., Messer, C. J., Chew, A., Han, J.-H. and others (2001), Haplotype variation and linkage disequilibrium in 313 human genes, Science 293, 489-493.

Sullivan, P. F., Daly, M. J., and O'donovan, M. (2012), Genetic architectures of psychiatric disorders: the emerging picture and its implications, Nature Reviews Genetics 13, 537551.

Tenesa, A.and Haley, C. S. (2013), The heritability of human disease: estimation, uses and abuses, Nature Reviews Genetics 14, 139-149.

van Rheenen, W., Peyrot, W. J., Schork, A. J., Lee, S. H., and Wray, N. R. (2019), Genetic correlations of polygenic disease traits: from theory to practice, Nature Reviews Genetics $20,567-581$. 


\section{REFERENCES}

Wang, J. and Li, H., (2021), Estimation of genetic correlation with summary association statistics, Biometrika.

Yang, J., Benyamin, B., McEvoy, B. P., Gordon, S., Henders, A. K., Nyholt, D. R., Madden, P. A., Heath, A. C., Martin, N. G., Montgomery, G. W. and others (2010), Common SNPs explain a large proportion of the heritability for human height, Nature Genetics 42, 565-569.

Yang, J., Lee, S.H., Goddard, M.E. and Visscher, P.M., (2011), GCTA: a tool for genome-wide complex trait analysis, The American Journal of Human Genetics, 88(1), pp.76-82.

Zhang, Y., Cheng, Y., Jiang, W., Ye, Y., Lu, Q. and Zhao, H., (2021), Comparison of methods for estimating genetic correlation between complex traits using GWAS summary statistics, Briefings in bioinformatics, 22(5), p.bbaa442.

Zhao, B. and Zhu, H., (2021), On genetic correlation estimation with summary statistics from genome-wide association studies, Journal of the American Statistical Association, pp.1-11.

Zheng, J., Erzurumluoglu, A. M., Elsworth, B. L., Kemp, J. P., Howe, L., Haycock, P. C., Hemani, G., Tansey, K., Laurin, C., Pourcain, B. S. and others (2017), LD Hub: a centralized database and web interface to perform LD score regression that maximizes the potential of summary level GWAS data for SNP heritability and genetic correlation analysis, Bioinformatics 33, 272-279.

Department of Statistics, University of California, 399 Crocker Lane, Davis, CA 95616, USA. 


\section{REFERENCES}

E-mail: jimjiang@ucdavis.edu

Department of Biostatistics, Yale School of Public Health, 60 College Street, New Haven, CT 06510, USA.

E-mail: w.jiang@yale.edu

Department of Statistics, University of California, 399 Crocker Lane, Davis, CA 95616, USA.

E-mail: debpaul@ucdavis.edu

Department of Biostatistics, Yale School of Public Health, 60 College Street, New Haven, CT 06510, USA.

E-mail: yiliang.zhang@yale.edu

Department of Biostatistics, Yale School of Public Health, 60 College Street, New Haven, CT 06510, USA.

E-mail: hongyu.zhao@yale.edu 